\title{
SPECT/CT: Looking Beyond Sentinel Lymph Node Identification for Improving Patient Outcomes
}

\author{
Robert Sibley, MD ${ }^{1}$ and Rathan M. Subramaniam, MD, PhD, MPH ${ }^{1,2}$ \\ ${ }^{1}$ Division of Nuclear Medicine, Department of Radiology, University of Texas Southwestern Medical Center, Dallas, TX; \\ ${ }^{2}$ Harold Simmons Comprehensive Cancer Center, University of Texax Southwestern Medical Center, Dallas, TX
}

Sentinel lymph node (SLN) identification is required for accurate prognostication for patients with head and neck melanoma. ${ }^{1}$ In clinical practice, identifying SLNs can be challenging. This is particularly true for patients with head and neck melanomas, which are associated with higher rates of false negative biopsies. ${ }^{2}$ Additionally, the drainage pattern of the head and neck is complex. Drainage patterns can be discordant from predicted patterns, ${ }^{3,4}$ and a single lesion can drain to multiple basins. ${ }^{4-7}$ Unfortunately, these challenges have resulted in a relative paucity of research for SLN studies in patients with head and neck melanoma.

Planar sentinel lymphoscintigraphy is a highly functional and sensitive test to demonstrate lymphatic channels, and to identify SLNs and nodes with microscopic nodal metastasis. It provides valuable information about individual lymphatic collectors reaching the SLN, dynamic arrival time of the radiotracer at the SLN, number of lymphatic collectors leaving the melanoma site, and relative intensity of lymph nodes; however, it lacks information about detailed anatomy that would be helpful in surgical planning and identifying sentinel nodes closer to the injection site due to blooming artifact. Single photon emission computed tomography/computed tomography (SPECT/CT) overcomes this disadvantage and provides high-resolution anatomic images to localize the SLNs and second-tier lymph nodes. It has been demonstrated that SPECT/CT not only increases detection of metastatic involvement ${ }^{8}$ but has also been associated with significant cost reduction. ${ }^{9}$

\footnotetext{
(C) Society of Surgical Oncology 2017
}

First Received: 23 November 2017;

Published Online: 7 December 2017

R. M. Subramaniam, MD, PhD, MPH

e-mail: rathan.subramaniam@UTsouthwestern.edu
Trinh and colleagues performed a retrospective review ${ }^{10}$ of 118 patients undergoing an SLN biopsy for head and neck cutaneous melanoma, all of whom underwent a SPECT/CT. Patients were categorized into two groups: ${ }^{1}$ those in whom SPECT/CT did not identify additional nodal basins; and $^{2}$ those in whom SPECT/CT did identify additional basins. Two radiologists interpreted all the imaging studies and answered questions regarding the utility of SPECT/CT in addition to planar imaging. SLNs were then identified on the basis of radioactivity and/or dye uptake, and biopsied. The surgeons performing the procedure answered a survey regarding the impact of SPECT/CT for the extent of SLN biopsy incision and utility in detecting the SLNs. Long-term outcomes were then described. Of the patients with positive lymph node disease, $10 \%(2 / 20)$ were missed by planar imaging alone. The survey results suggest that SPECT/CT influenced the extent of the incision and helped to localize a SLN close to the primary tumor. In addition, the study by Trinh et al. showed that the sites of locations of SLNs where SPECT/CT is most helpful are the supraclavicular and peri-parotid regions. SPECT/CT added little information in identifying new SLNs in other neck levels.

The outcomes addressed in their study, including disease-free survival, overall survival, locoregional recurrence, and distant recurrence, are of utmost importance to patients and clinicians. It is important that future prospective studies establish the benefits of SPECT/CT (i.e., identification of additional SLNs at the supraclavicular and peri-parotid locations, and influencing the extent of incision or surgical planning) and impacts the outcome of patients, especially quality of life and survival outcomes. SPECT/CT may offer benefit to improve quality-of-life outcomes by reducing complication rates and comorbidities. The overall complication rate for SLN biopsy for melanoma was $11.3 \%$, and the incidence of infection was 
$2.9 \%$, seroma $5.1 \%$, hematoma $0.5 \%$, lymphedema $1.3 \%$, and nerve injury $0.5 \% .^{11}$ Patients with melanoma who undergo complete lymph node dissection (CLND) have a $37.2 \%$ complication rate, including wound infection/ breakdown (21\%), lymphedema (18\%), and seroma $(18 \%){ }^{12}$ It is likely these complications were factors in many patients refusing CLND. Planar lymphoscintigraphy with SPECT/CT not only localizes lymph nodes but also identifies lymphatic vessels. Avoiding major lymphatic vessels hypothetically reduces surgical complications, in addition to reducing incision length. If the data were to support utility for localizing lymphatic vessels, this could have broad implications in future.

Trinh et al. have excelled in addressing the potential utility of SPECT/CT for patients with melanoma in head and neck cancer patients, however more research remains to be conducted to demonstrate its utility and impact on quality of life and survival outcomes for patients.

\section{REFERENCES}

1. Monroe MM, Pattisapu P, Myers JN, Kupferman ME. Sentinel lymph node biopsy provides prognostic value in thick head and neck melanoma. Otolaryngol Head Neck Surg. 2015;153(3): 372-78.

2. Lee DY, Huynh KT, Teng A, et al. Predictors and survival impact of false-negative sentinel nodes in melanoma. Ann Surg Oncol. 2016;23(3):1012-18.

3. Lin D, Franc BL, Kashani-Sabet M, Singer MI. Lymphatic drainage patterns of head and neck cutaneous melanoma observed on lymphoscintigraphy and sentinel lymph node biopsy. Head Neck. 2006;28(3):249-55.
4. Kaveh AH, Seminara NM, Barnes MA, et al. Aberrant lymphatic drainage and risk for melanoma recurrence after negative sentinel node biopsy in middle-aged and older men. Head Neck. 2016;38(Suppl 1):E754-60.

5. Vidal M, Vidal-Sicart S, Torres F, Ruiz DM, Paredes P, Pons F. Correlation between theoretical anatomical patterns of lymphatic drainage and lymphoscintigraphy findings during sentinel node detection in head and neck melanomas. Eur J Nucl Med Mol Imaging. 2016;43(4):626-34.

6. Bostick P, Essner R, Sarantou T, et al. Intraoperative lymphatic mapping for early-stage melanoma of the head and neck. Am J Surg. 1997;174(5):536-39.

7. Stewart CL, Gleisner A, Kwak J, et al. Implications of sentinel lymph node drainage to multiple basins in head and neck melanoma. Ann Surg Oncol. 2017;24(5):1386-91.

8. Jimenez-Heffernan A, Ellmann A, Sado H, et al. Results of a prospective multicenter international atomic energy agency sentinel node trial on the value of SPECT/CT over planar imaging in various malignancies. J Nucl Med. 2015;56(9):1338-44.

9. Stoffels I, Muller M, Geisel MH, et al. Cost-effectiveness of preoperative SPECT/CT combined with lymphoscintigraphy versus lymphoscintigraphy for sentinel lymph node excision in patients with cutaneous malignant melanoma. Eur J Nucl Med Mol Imaging. 2014;41(9):1723-31.

10. Trinh BB, CB, Gleisner A, Kwak JJ, Morgan R, McCarter MD, Gajdos C, Kounalakis N. SPECT/CT adds distinct lymph node basins and influences radiologic findings and surgical approach for sentinel lymph node biopsy in head and neck melanoma. Ann Surg Oncol. In press.

11. Moody JA, Ali RF, Carbone AC, Singh S, Hardwicke JT. Complications of sentinel lymph node biopsy for melanoma: a systematic review of the literature. Eur J Surg Oncol. 2017;43(2):270-77.

12. Moody JA, Botham SJ, Dahill KE, Wallace DL, Hardwicke JT. Complications following completion lymphadenectomy versus therapeutic lymphadenectomy for melanoma: a systematic review of the literature. Eur J Surg Oncol. 2017;43(9):1760-67. 